\title{
Clinical Safety Assessment of Infant Nutrition
}

\author{
M.S. Fewtrell \\ Childhood Nutrition Research Centre, UCL Institute of Child Health, London, UK
}

\author{
Key Words \\ Infant nutrition - Randomized trial · Safety • Efficacy • \\ Outcomes $\cdot$ Follow-up · Attrition
}

\begin{abstract}
Data on clinical safety and efficacy are ideally collected in a randomized clinical trial or, failing this, an observational study. Suitable outcomes vary depending on the intervention and population group, and certain outcomes such as growth may test both efficacy and safety. The use of growth as an important safety outcome has some limitations since it is currently not clear what represents an 'optimal' growth pattern. Several issues currently make the conduct and interpretation of infant nutrition trials challenging. These include difficulties in recruiting exclusively formula-fed infants, particularly given the emotive nature of infant feeding; the involvement of industry leading to real or perceived conflicts of interest; increased regulation and bureaucracy; and particular issues with long-term follow-up studies, notably cohort attrition. This paper addresses the implications of these issues and some potential solutions.
\end{abstract}

Copyright ๑ 2012 S. Karger AG, Basel

Clinical safety assessment in infant nutrition is generally performed in the context of a clinical study or trial, which can be observational or experimental (randomized
(C) 2012 S. Karger AG, Basel

$0250-6807 / 12 / 0603-0200 \$ 38.00 / 0$

Fax +41613061234

E-Mail karger@karger.ch

www.karger.com
Accessible online at:

www.karger.com/anm controlled trial, RCT). Observational studies can demonstrate associations between the nutritional intervention and later outcome but cannot establish causality, whereas randomized trials are regarded as the 'gold standard' in terms of demonstrating causality and underpinning clinical practice. The 'pharmaceutical-style' approach of using RCTs to test safety and efficacy was first applied in the field of infant nutrition in the 1980s [1] and is now accepted as the preferred approach for evaluating novel nutritional interventions, including, notably, the modification of or addition of novel ingredients to breast milk substitutes.

Over the past 3 decades, there has also been a shift in the focus of research in infant nutrition from meeting nutritional requirements and avoiding deficiency states towards optimizing clinical outcomes, both short-term and long-term. This approach necessitates identifying appropriate outcome measures for both safety and efficacy, and also introduces the additional challenges of longer-term follow-up of study participants [2].

\section{Choice of Safety Outcomes}

The most appropriate safety outcome will vary depending on the population group and intervention, and it is sometimes difficult to distinguish whether an outcome is primarily measuring safety or efficacy. In studies in- 
volving preterm infants, common short-term clinical safety outcomes (more often referred to as 'adverse outcomes') include indicators of sepsis, feed tolerance, and necrotizing enterocolitis or death, as well as biochemical and hematological measurements [3, 4]. Indicators of feed tolerance may also be used in healthy term infants, together with infection or atopy [5]. However, perhaps the most widely used indicator of safety is infant growth - and this is frequently also used as a measure of efficacy [3-6]. Although the general concept that infants receiving a new intervention should broadly show a 'similar' pattern of growth to infants receiving an existing form of nutrition seems reasonable, the concept that an intervention is 'safe' provided the average growth of study infants does not differ by more than 0.5 standard deviations (SD) compared to a reference or control group is open to debate, since the optimal growth pattern during infancy is not certain. Whereas in the past, concern would most likely have been generated if an intervention group showed slower growth than the control group, with recent focus on the potential disadvantages of more rapid infant growth for later cardiovascular health and obesity [7-12], this perspective has changed; an intervention that results in slower growth than seen in a control group might now be considered beneficial. The conclusions drawn from any growth comparison will also depend on the reference group used - for example, breast-fed infants or those fed a 'standard' formula - and also on the growth reference used to generate standardized (indicated in SD) growth scores. Although widely discussed and used to determine or justify sample size in randomized nutritional intervention trials, it is important to appreciate that the selection of $0.5 \mathrm{SD}$ as the effect size required to establish or exclude a biologically or clinically important difference between groups is essentially arbitrary and also open to debate.

\section{Randomized Trials in Infant Nutrition - Practical Issues}

Although accepted as the gold standard for evaluating any novel nutritional intervention, there are a number of issues regarding the design, implementation and evaluation of RCTs in the field of infant nutrition.

Design

Healthy term infants cannot be ethically or feasibly randomized to be breast-fed or formula-fed, thus precluding (in most situations) the use of a RCT for assessing the safety of an intervention against the 'gold standard'

Clinical Safety Assessment of Infant

Nutrition for infant feeding. Consequently, the clinical safety testing of new interventions generally involves a randomized comparison between infants who receive the intervention (for example, a supplemented formula) versus those who receive the 'standard' diet (for example, an unsupplemented formula). It is considered good practice to include a reference group of breast-fed infants, who follow the same protocol as far as possible, but without randomization, necessitating adjustment for confounding factors in any subsequent comparison of outcomes with those of the formula-fed randomized groups.

In current practice, given the emotive nature of infant feeding, recruiting and randomizing formula-fed infants shortly after birth, without jeopardizing (or, perhaps more importantly, being seen to jeopardize) breastfeeding can be challenging. The key issue is at what point a mother can be considered to have unequivocally decided not to breastfeed, making her eligible for randomization to a breast milk substitute, particularly in the situation where a free supply of the study formula is provided. Many study protocols, particularly those involving healthy term infants, require infants to be exclusively formula-fed to be eligible for randomization, to avoid compromising any ongoing breastfeeding. With increasing rates of 'mixed' breast- and formula-feeding early in the postnatal period, recruitment of exclusive formula feeders has become ever more challenging in some settings.

\section{Role of Industry}

Many, if not the majority, of clinical safety assessments in infant nutrition involve products developed and produced by companies that manufacture infant formulas. The majority of these studies also receive financial support from the company, although the precise nature of the support can vary. At one extreme, the company may provide a research grant to support the study plus a supply of the product being tested, with little or no involvement in the study conduct, data analysis or subsequent dissemination of data. At the other extreme (less common in the field of infant nutrition but perhaps more so in pharmaceutical research), the company may essentially commission a research group to conduct the study, maintaining day-to-day control over the conduct of the study, data analysis and write-up. Industry involvement naturally raises the issue of conflict of interest, and introduces yet more controversy to an already emotive field.

A further issue in the relationship between academics and industry relates to time scales, which frequently differ for fundamental parameters such as planning and conducting research studies and publishing or releasing data. 
Table 1. Suggestions for resolving difficulties in conducting infant nutrition research studies and trials

\begin{tabular}{|c|c|}
\hline Problem & Suggested solution(s) \\
\hline $\begin{array}{l}\text { Lack of clear guidance on requirements } \\
\text { establishing safety and efficacy }\end{array}$ & $\begin{array}{l}\text { Greater consensus and guidance on study requirements including suitable safety for } \\
\text { outcomes, sample sizes, number of studies required or number of subjects to be studied. }\end{array}$ \\
\hline $\begin{array}{l}\text { Lack of consistency in study outcomes } \\
\text { and methodology }\end{array}$ & $\begin{array}{l}\text { Greater standardization of data collection and outcome measures between studies. The use } \\
\text { of a common data set - including the outcomes to be measured and how they are } \\
\text { measured (proposed in a position paper by the ESPGHAN committee on nutrition in } 2001 \\
{[14] \text { ) would facilitate systematic reviews and meta-analyses. }}\end{array}$ \\
\hline $\begin{array}{l}\text { Conflict of interest with industry } \\
\quad \text { (real or perceived) }\end{array}$ & $\begin{array}{l}\text { More explicit reporting of the details of these relationships at the time a study is published. } \\
\text { More explicit support and guidance from funding bodies and universities for acceptable } \\
\text { working relationships between academics and industry might also reduce the perception } \\
\text { that such associations are shameful or inherently flawed. }\end{array}$ \\
\hline $\begin{array}{l}\text { Time-consuming and complicated } \\
\text { regulatory processes }\end{array}$ & Simplify the regulatory processes required to set up and conduct trials. \\
\hline
\end{tabular}

\section{Regulatory Aspects}

Clinical research has become ever more time consuming in recent years with increasing requirements for regulatory approvals, and frequent changes in requirements over short periods of time. Although requirements vary between countries, in most settings this is now perceived as a significant issue by researchers and, in some cases, represents a disincentive to conducting new studies.

\section{Long-Term Follow-Up}

Although the later health effects (both good and bad) of early nutrition are generally acknowledged as important outcomes and long-term follow-up is encouraged, this introduces an additional set of problems, including cohort attrition with time [2]. This is recognized to be a problem in both RCTs and observational studies. Loss to follow-up varies depending on a number of factors including the duration of follow-up, population, invasiveness of proposed tests, and degree of inconvenience to the subject. The statistical consequences of attrition - mainly effects on study power, bias, and generalizability - have been recently reviewed, with proposals for how these issues might best be addressed and discussed when the results of these studies are published [2]. The alternative - setting fixed levels for what constitutes an 'acceptable' follow-up rate (for example, $80 \%$ used to indicate a highquality study for evidence-based medicine [13]) - is likely to render many long-term studies non-viable, since they may effectively be unpublishable.

\section{The Future}

Suggestions aimed at resolving some of the issues raised above are given in table 1; most are not new, and the fact that they have not, to date, been realized, perhaps indicates the difficulty of implementing seemingly sensible ideas in practice. However, action is certainly required if high-quality clinical efficacy and safety data are to be collected in future in this field.

\section{Disclosure Statement}

Dr. Fewtrell has received research funding and performed consultancy work for companies manufacturing infant formula and infant feeding products. She is a member of the RCPCH Nutrition Committee (UK) and the ESPGHAN Committee on $\mathrm{Nu}-$ trition. She has also been a member of the National Childbirth Trust (NCT; UK) and been involved in breastfeeding counseling. 


\section{References}

$>1$ Lucas A, Gore SM, Cole TJ, et al: A multicentre trial on the feeding of low birthweight infants: effects of diet on early growth. Arch Dis Child 1984;59:722-730.

$>2$ Fewtrell MS, Kennedy K, Singhal A, Martin RM, Ness A, Hadders-Algra M, Koletzko B, Lucas A: How much loss to follow-up is acceptable in long-term randomised trials and prospective studies? Arch Dis Child 2008;93: 458-461.

3 AlFaleh K, Anabrees J, Bassler D, Al-Kharfi T: Probiotics for prevention of necrotizing enterocolitis in preterm infants. Cochrane Database Syst Rev 2011;3:CD005496. DOI: 10.1002/14651858.CD005496.pub3.

4 Kuschel CA, Harding JE: Multicomponent fortified human milk for promoting growth in preterm infants. Cochrane Database Syst Rev 2004;1:CD000343. DOI: $10.1002 /$ 14651858.CD000343.pub2.

$>5$ Lucas A, Stafford M, Morley R, Abbott R, Stephenson T, MacFadyen U, Elias-Jones A, Clements H: Efficacy and safety of longchain polyunsaturated fatty acid supplementation of infant-formula milk: a randomised trial. Lancet 1999;354:1948-1954.
6 Fewtrell MS, Abbott RA, Kennedy K, Singhal A, Morley R, Caine E, Jamieson C, Cockburn F, Lucas A: Randomized, double-blind trial of long-chain polyunsaturated fatty acid supplementation with fish oil and borage oil in preterm infants. J Pediatr 2004;144: 471-479.

7 Monteiro POA, Victora CG: Rapid growth in infancy and childhood and obesity in later life - a systematic review. Obes Rev 2005;6: 143-154.

8 Ong KK, Loos RJF: Rapid infancy weight gain and subsequent obesity: systematic re views and hopeful suggestions. Acta Paediatr 2006;95:904-908.

$\checkmark 9$ Stettler N: Nature and strength of epidemiological evidence for origins of childhood and adult obesity in the first year of life. Int J Obes 2007;31:1035-1043.
10 Singhal A, Cole TJ, Fewtrell M, Kennedy K, Stephenson T, Elias-Jones A, Lucas A: Promotion of faster weight gain in infants born small for gestational age: is there an adverse effect on later blood pressure? Circulation 2007;115:213-220.

$>11$ Ben-Shlomo Y, McCarthy A, Highes R, Tilling K, Davies D, Smith GD: Immediate postnatal growth is associated with blood pressure in young adulthood: The Barry Caerphilly Growth Study. Hypertension 2008;52: 638-644.

12 Singhal A, Cole TJ, Fewtrell M, Deanfield J, Lucas A: Is slower early growth beneficial for long-term cardiovascular health? Circulation 2004;109:1108-1113.

13 Oxford CEBM: http://www.cebm.net/index. aspx?o=1025 (accessed May 13, 2011).

14 Aggett PJ, Agostini C, Goulet O, Hernell O, Koletzko B, Lafeber HL, Michaelsen KF, Rigo J, Weaver LT: The nutritional and safety assessment of breast milk substitutes and other dietary products for infants: a commentary by the ESPGHAN Committee on Nutrition. J Ped Gastroenterol Nutr 2001;32: 256-258. 